\title{
THE USE OF CONTINUED FRACTIONS IN THE DESIGN OF ELECTRICAL NETWORKS*
}

\author{
BY T. C. FRY
}

1. Introduction. The literature on the subject of electrical circuit theory deals very largely with one type of problem: "Given an electrical system and the driving force which is imposed upon it, to find how it responds."

The converse problem, namely "Given a known driving force and the response which it is desired to produce, to find a system which will so respond," is much less frequently dealt with in the literature. It is this problem with which the present paper is to deal. $\dagger$

About the electrical side of the problem we need only say that, whatever the language in which the engineer may phrase his requirements, they can always be satisfied provided we can produce one or more networks of preassigned impedances. It is, then, no serious restriction upon the prac-

* An address presented at the request of the program committee at the meeting of this Society at Cincinnati, December 1, 1928.

$\dagger$ Those who are interested in following the earlier literature will find the following references helpful. They will also give some idea of the technical problems to which the theory is applicable.

Fry, U. S. Patent \#1,570,215, 1926, filed June 1921.

Zobel, Theory and design of uniform and composite electric wave filters, Bell System Technical Journal, vol. 2 (1923), p. 1.

Bartlett, A note on the theory of artificial telephone and transmission lines, Philosophical Magazine, vol. 48 (1924), p. 859.

Bartlett, Properties of the generalized artificial line, Philosophical Magazine, vol. 1 (1926), p. 553.

Cauer, Die Verwirklichung von Wechselstromwiderständen vorgeschriebener Frequenzabhängigkeit, Archiv für Elektrotechnik, vol. 17 (1926), pp. $355-388$.

Bartlett, British Patent \#290,701, 1928, filed January 1927.

Mead, Phase distortion and phase distortion correction, Bell System Technical Journal, vol. 7 (1928), p. 195.

Zobel, Distortion correction in electrical networks with constant resistance recurrent networks, Bell System Technical Journal, vol. 7 (1928), p. 438. 
ticability of our theory from a technical standpoint to confine ourselves entirely to the design of networks with given impedance properties.

Our point of departure is a very simple observation regarding the ladder-type network shown in Fig. 1. The ordinary laws of circuit theory lead immediately to the formula*

$$
F(p)=J_{1}+\frac{1}{Y_{2}+\frac{1}{J_{3}+\frac{1}{Y_{4}+\cdots}} .} .
$$

If, then, the various $J$ 's and $Y$ 's are known, we can write down $F(p)$ at once. For example, if all the series elements

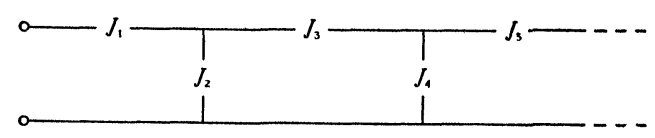

FIG. 1

are resistances $r_{1}, r_{3}, \cdots$, and all the shunt elements capacities $c_{2}, c_{4}, \cdots$, (1) will have the form of the Stieltjes fraction

$$
F(p)=r_{1}+\frac{1}{c_{2} p+\frac{1}{r_{3}+\cdots}} .
$$

Conversely, if we are given the problem of designing a network the impedance of which is to be some known function of frequency, and if we can expand this function $F(p)$ in a continued fraction of the Stieltjes type, we can certainly furnish the required network; for we need only cause the successive resistances in our ladder structure to be equal to the successive constant terms of the fraction, and the successive capacities to the coefficients of the corresponding terms in $p$. $J_{\boldsymbol{i}}$.

* The admittances $Y_{i}$ are reciprocals of the corresponding impedances 
We can, then, apply the entire theory of Stieltjes fractions directly to the problem of network design. But there are many physical networks-even many networks of the ladder type-whose impedances cannot be expanded in the form (2), though they can be expressed in the form (1) if the $J$ 's and $Y$ 's are not restricted to be constants and multiples of $p$. For example, the simple function $\dagger$

$$
F^{*}(p)=\frac{2 p+3}{p+2}
$$

cannot be represented by a Stieltjes fraction, though it can be written in the form

$$
F^{*}(p)=1+\frac{1}{1+\frac{1}{p+1}},
$$

which is quite suitable for purposes of design. The Stieltjes fraction (2), in other words, is too special for our needs.

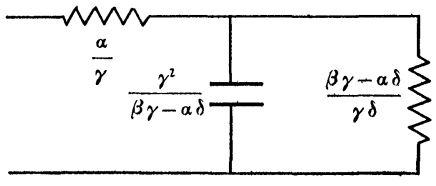

FIG. 2

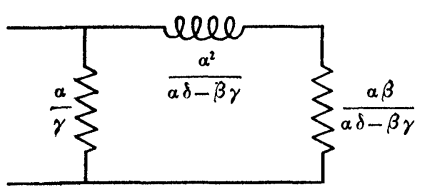

FIG. 4

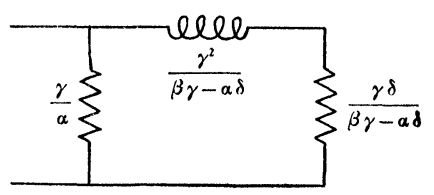

Fig. 3

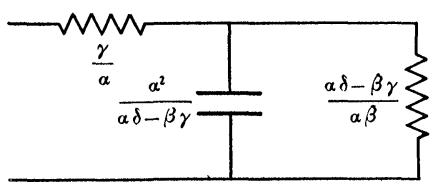

FIG. 5

In the present paper we shall obtain the necessary degree of generality by allowing the $J$ 's and $Y$ 's of (1) to be of the bilinear form

$$
\frac{\alpha p+\beta}{\gamma p+\delta}
$$

† The reason for the asterisk will appear later. 
$\alpha, \beta, \gamma$ and $\delta$ being positive constants. Such a bilinear form can always be assigned a definite design significance. For if $\beta \gamma>\alpha \delta$, it can be interpreted as the impedance of the structure shown in Fig. 2 and the admittance of that shown in Fig. 3; while if $\beta \gamma<\alpha \delta$, it can be interpreted as the impedance of Fig. 4, or the admittance of Fig. 5.

2. Resumé of Theorems about Stieltjes Fractions. Obviously, since the Stieltjes fraction is so closely related to our problem, we must have the essential facts concerning it before us. They are as follows.

Theorem 1. A Stieltjes fraction

$$
f(z)=\frac{1}{a_{1} z+\frac{1}{a_{2}+\frac{1}{a_{3} z+\cdots}}}
$$

converges for every value of the complex variable $z$, except perhaps certain negative real values, provided the a's are positive reals, and provided $\sum a_{j}$ diverges.*

THEOREM 2. The function $f(z)$ defined by a convergent Stieltjes fraction can always be expressed in the form

$$
f(z)=\int_{0}^{\infty} \frac{d \Phi(x)}{z+x},
$$

where $\Phi(x)$ is a monotonic non-decreasing real function of the real variable $x$, where $\Phi(0)=0$ and $\Phi(\infty)=1 / a_{1}$. It is not necessary that $\Phi(x)$ be continuous. $\dagger$

* Stieltjes, CEuvres Complètes, vol. 2, p. 465. If $\Sigma a_{j}$ converges, the convergents of even and odd orders separately approach limits $f_{1}(z)$ and $f_{2}(z)$, but the limits are not equal. As we have no interest in this case we shall give no further attention to it.

The case of complex $a$ 's was afterward discussed by Van Vleck, Transactions of this Society, vol. 2 (1901), pp. 262, 344. Such coefficients have no meaning in the problem of network design, and will therefore not be considered here.

† Stieltjes, loc. cit., pp. 491, 493. 
THEOREM 3. The function $f(z)$ defined by a convergent Stieltjes fraction is therefore a regular analytic function, except at certain points of the negative real axis,* and is real for positive real values of $z$. The same is true of its reciprocal. $†$

Theorem 4. The function $\Phi(x)$ in (5) is related to $f(z)$ by the law

$$
\Phi(x)=\frac{1}{2 \pi i} \int f(z) d z,
$$

the path of integration extending along a circle of radius $x$ about the origin, beginning at $-x-i 0$ and ending at $-x+i 0 . \ddagger$

THeOrem 5. Conversely, if $\Phi(x)$ is any monotonic nondecreasing real function of $x$ in the "finite" interval $(0, b)$, and constant for $x \geqq b$, the function $f(z)$ defined by (5) possesses a convergent Stieltjes expansion with positive real coefficients. $\S$

Theorem 6. If $\Phi(x)$ is any monotonic non-decreasing function of $x$, such that the integrals

$$
\int_{0}^{\infty} x^{n} d \Phi(x)
$$

all exist, the function $f(z)$ defined by (5) possesses a "formal" Stieltjes expansion with positive real coefficients, which, if it converges at all, converges to the value $f(z)$ for all values of $z$ except those on the negative real axis.\|

* Perron, Die Lehre von den Kettenbrüchen, p. 369.

$\dagger$ This is readily seen as follows: If (4) converges to $f(z)$, the fraction

$$
\frac{1}{a_{2} x+\frac{1}{a_{3}+\cdots}}
$$

must converge to $f_{1}(z)=1 /[z f(z)]-a_{1}$; for the convergents of this latter fraction are the corresponding convergents of the former one, divided by $z$ and reduced by $a_{1}$. The new fraction, however, is a Stieltjes fraction, and therefore $f_{1}(z)$, and hence also $1 / f(z)$, is regular everywhere except on the negative real $z$-axis.

$\ddagger$ This is a slight modification of the theorem as given by Perron, loc. cit., p. 372.

$\S$ Perron, loc. cit., p. 388.

\| Stieltjes, loc. cit., p. 504. 
Thus, if we are given a function $f^{*}(z)$ we can determine whether or not it possesses a Stieltjes expansion by the following systematic procedure:

(a) First we observe whether it is real for positive real values of $z$. If not, no usable expansion is possible.

(b) Next we investigate the singularities of $f^{*}(z)$ and its reciprocal. Unless they are all on the negative real axis no expansion is possible.

(c) If they are all on the negative real axis we compute a function $\Phi(x)$ from the formula

$$
\Phi(x)=\frac{1}{2 \pi i} \int f^{*}(z) d z,
$$

the path of integration being that defined in Theorem 4 . Unless it is monotonic non-decreasing, no expansion is possible. Even if it is monotonic non-decreasing no expansion is possible unless either $\Phi(x)$ is a finite constant for $x>b$, or else the integrals (7) all exist.

(d) If the possibility of expansion is still not disproved, we substitute our $\Phi(x)$ in (5) and evaluate the integral. The $f(z)$ which results may or may not be the function $f^{*}(z)$ with which we started. If it is not, $f^{*}(z)$ can neither be expressed in the form (5), nor expanded in a Stieltjes fraction. But if $f(z)$ is identical with $f^{*}(z)$, we know either that a convergent expansion does exist, or that it may exist, according as we are working under the conditions of Theorems 5 or $6 . \dagger$

We may, then, fail to get a Stieltjes expansion for either of four reasons:

(a) Because $f^{*}(z)$ is not real when $z$ is real and positive;

† We say nothing about the processes by means of which the coefficients are obtained after we have shown that an expansion is possible, for the reason that these processes play no part in our study. They are tedious, but otherwise quite satisfactory. They will be found in almost any discussion of continued fractions, the most satisfactory references being Stieltjes, Euvres Complètes, vol. 2, p. 184, for the more familiar method, and Thiele, Interpolationsrechnung, p. 138, for an interesting alternative. 
(b) Because the singularities of $f^{*}(z)$ or its reciprocal are not properly located;

(c) Because $\Phi(x)$ is not of an allowable type;

(d) Because, though $f^{*}(z)$ gives rise to a $\Phi(x)$ of suitable type, it is still not true that

is equal to $f^{*}(z)$.

$$
f(z)=\int \frac{d \Phi(x)}{z+x}
$$

We shall consider these difficulties in order, seeking in each case to find a means of deriving a type of fraction which can be interpreted electrically.

We shall occasionally find it desirable to have separate names for the functions $f^{*}(z)$ and $f(z)$. The former of thesethat is, the function which we wish to represent in fractional form, and from which we obtain $\Phi(x)$ by the use of (6)-we shall call the generating function. The other-that is, the function to which the fraction converges if we succeed in getting a convergent fraction at all, and which is obtained from $\Phi(x)$ by the use of (5) - will be said to correspond to $\Phi(x)$. The important distinction is, that while many functions may generate the same $\Phi(x)$, only the one which corresponds to it is capable of Stieltjes representation.

3. Redistribution of Singularities by Change of Variable. The first difficulty is inherent in the physical problem. For if a physical system had an impedance $Z(p)$ which was complex for a real positive $p=p_{0}$, a real driving force which varied with time according to the law $e^{p_{0} t}$ would produce a complex current. This is absurd, since a complex current has no physical meaning.

Turning next to the second difficulty, we first note that physically possible systems cannot have singularities in the right-hand half of the plane. This is easily seen in the case of poles, for a pole would imply that once an oscillation of the proper frequency were established it would persist forever with constantly increasing amplitude even in the absence of any sustaining force, which is obviously absurd. 
It is not so easy to exclude the possibility of essential singularities, but they are never met.

On the other hand, singularities in the left-hand half of the plane-or as a limiting case on the imaginary axisare physically possible. In some simple cases they can be shifted to the negative real axis by a suitable change in variable, and an expansion thus obtained.

Thus, suppose we are given a function $F^{*}(p)$ which, together with its reciprocal, has only pure imaginary singular points. Then by the transformation

$$
z=p^{2}
$$

we will arrive at a function $f^{*}(z) \equiv F^{*}\left(z^{1 / 2}\right)$, whose singularities occur at negative real values of $z$. From this function we may be able to obtain a Stieltjes fraction of the form (4), and if so the equivalent fraction in $p$,

$$
F(p) \equiv f\left(p^{2}\right)=\frac{1}{a_{1} p^{2}+\frac{1}{a_{2}+\cdots}},
$$

will converge for all values of $p$ except possibly certain pure imaginaries.

Even though this $F(p)$ proved to be identical with $F^{*}(p)$, however, it would not be suitable for the purposes of circuit design; for we have no type of circuit element whose impedance or admittance varies as the square of the frequency. However, if we were to set $f^{*}(z) \equiv F^{*}\left(z^{1 / 2}\right) / z^{1 / 2}$, we might arrive at a usable expansion. $\dagger$ For suppose we succeeded in finding a convergent fraction (4) the limit of which was identical with this $f^{*}(z)$. We would then have at once

$$
F^{*}(p)=\frac{1}{a_{1} p+\frac{1}{a_{2} p+\frac{1}{a_{3} p+\cdots}}} .
$$

$\dagger$ We have probably introduced a new singularity at $z=0$ by the introduction of the new factor, but as this is an end point of the negative real axis, it is not objectionable. 
This form of development is altogether satisfactory. It corresponds to a ladder network all the series elements of which are inductances and all the shunt elements condensers.

Similarly, if the singularities of $F^{*}(p)$ and its reciprocal all lie on a line parallel to the imaginary axis and $\alpha$ units to the left of it, we may transfer them all to the negative real axis by either of the transformations $\dagger$

$$
z=(p+\alpha)^{2}
$$

or

$$
z=p^{2}+2 \alpha p .
$$

It is easily seen that either leads to a usable type of fraction provided we identify $f^{*}(z)$, not with $F^{*}(p)$, but rather with $F^{*}(p) /(p+\beta)$, where $0 \leqq \beta \leqq 2 \alpha . \ddagger$

4. Fractions which Represent Functions in Island Regions. The function $f^{*}(z)$ obtained from $F^{*}(p)$ by such transformations as (9), (10) and (11) is ordinarily multiple-valued.

On the other hand, the function defined by any fraction of the form (4) must be single-valued. Hence if we succeed in obtaining such a fraction, it will represent one branch of $f^{*}(z)$ only. Consequently, when rewritten in terms of $p$, it will represent $F^{*}(p)$ over only that portion of the $p$-plane which corresponds to this branch of $f^{*}(z)$. In the remainder of the $p$-plane it will converge (except, perhaps, at certain points of the boundary which separates the two regions), but usually not to the value $F^{*}(p)$.

The fraction, in other words, will represent $F^{*}(p)$ only in an "island region" (espace lacunaire). For example, by using the transformation $z=p^{2}+p$ we can expand the function (3) into the form

$\dagger$ The second of these transformations has a wider field of use than the first, for it carries to the negative real axis of $z$ not only the $p$ 's on the vertical line mentioned, but $p$ 's in the real range $(-2 \alpha, 0)$ as well. Singularities within this real interval are therefore also allowable.

$\ddagger$ Since $p=-\beta$ corresponds to a negative real $z$, the new singularity at this point is not objectionable. 


$$
F(p)=1+\frac{1}{\frac{2}{p+1}+\frac{1}{\frac{1}{p}+\frac{1}{\frac{1}{p+1}+\cdots}},}
$$

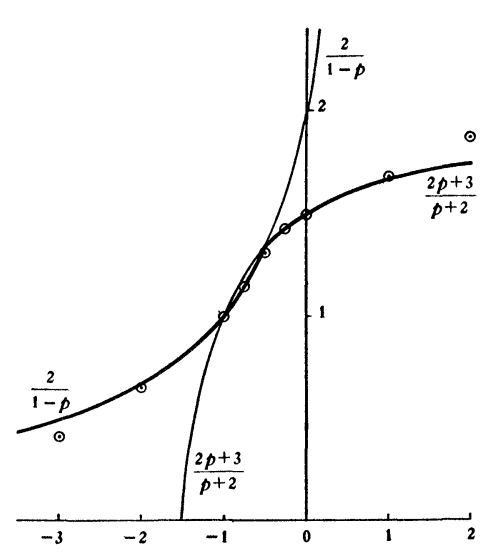

FIG. 6

the remaining terms being alternately $1 / p$ and $1 /(p+1)$. This fraction converges for every value of $p$, real or complex, except certain values with the real part $-1 / 2$. To the right of this dividing line the limit is actually equal to $F^{*}(p)=(2 p+3) /(p+2)$. To the left, it is $2 /(1-p)$. Fig. 6 shows the graphs of these functions for real values of $p$, together with certain points on the fifth convergent

of the fraction (12). It is obvious that, in the region covered by the graph, convergence is quite rapid.

Now the outstanding thing about this expansion is, that its singular line bears no relation to $F^{*}(z)$ whatever, but is determined by the character of the transformation instead. Had we begun with any other function and used the same transformation, and had we succeeded in obtaining a convergent fraction for this new function, the new fraction also would have had this same line for a cut, and would have been regular everywhere else.

5. Properties of a Particular Transformation. These observations at once suggest two methods of dealing with functions the singularities of which are not on the negative real axis. One is, to search for a transformation which will carry the singularities to the negative real axis. The other is, to search for a transformation which will carry them all 
into one branch of a multiple-valued function of $z$, thus leaving in the other branch only the singularities of the transformation itself. In either case, of course, we must require that the latter singularities shall lie on the negative real $z$-axis. The second of these methods is obviously the more powerful one, and we shall find that an especially suitable transformation is

$$
z=\frac{(a p+b)(\alpha p+\beta)}{(c p+d)(\gamma p+\delta)}
$$

where the coefficients $a, b, c, d, \alpha, \beta, \gamma, \delta$ are all positive reals.

It requires only routine algebra to establish the following facts :

(1) In order that the singular points of the transformation (13) shall be on the negative real $z$-axis it is necessary and sufficient that both

$$
\left\{\begin{array}{l}
(b \gamma-a \delta)(c \beta-d \alpha)>0, \\
(b c-a d)(\beta \gamma-\alpha \delta)>0 .
\end{array}\right.
$$

(2) The $p$-transformed of the negative real $z$-axis consists of part of the negative real $p$-axis together with a circle whose center is at

$$
p_{0}=-\frac{\beta \gamma(b c-a d)+a d(\beta \gamma-\alpha \delta)}{\alpha \gamma(b c-a d)+a c(\beta \gamma-\alpha \delta)},
$$

and whose radius is

$$
r^{2}=p_{0}^{2}-\frac{\beta \delta(b c-a d)+b d(\beta \gamma-\alpha \delta)}{\alpha \gamma(b c-a d)+a c(\beta \gamma-\alpha \delta)} .
$$

We shall call this circle the "boundary of the transformation."

(3) So long as (14) is satisfied, $p_{0}$ is negative and $r^{2} \leqq p_{0}^{2}$. Hence the boundary of the transformation never crosses the imaginary axis, though in a limiting case it may be tangent to it at the origin. It may also degenerate into a line parallel to, and to the left of, the imaginary axis; or in to the imaginary axis itself. We may therefore state the following theorem. 
THEOREM 7. Any transformation of the type (13) which has its singularities located on the negative real z-axis, and for which all the constants $a, b, c, d, \alpha, \beta, \gamma, \delta$ are positive or zero, maps a circular area in the left half of the p-plane upon one sheet of the z-plane, the remainder of the p-plane being mapped on the other sheet of the z-plane. The circular boundary may be tangent to the imaginary p-axis, or it may degenerate into a vertical straight line, but it cannot in any case enclose a portion of the right-hand half of the plane.

In view of these conclusions we can rewrite (13) in one or the other of three convenient forms.* The first is

$$
\begin{array}{ll}
z=\frac{r^{2} p^{2}}{\lambda^{2}(p+s)^{2}-p^{2}}, & s \geqq 0, \\
& \lambda \geqq 1,
\end{array}
$$

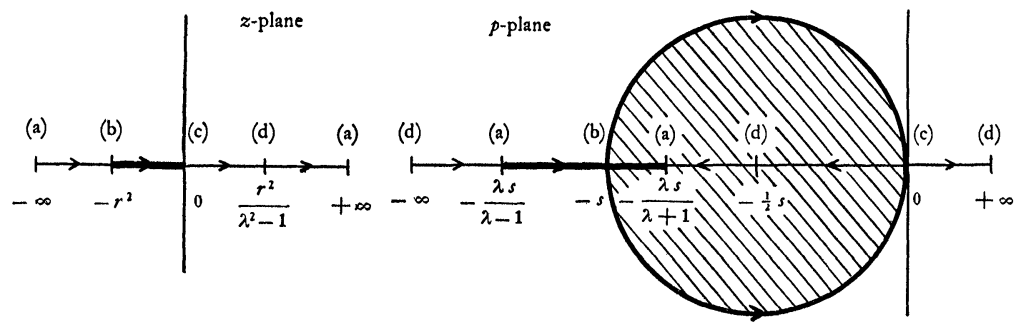

FIG. 7

which represents the configuration shown in Fig. 7. The boundary of the transformation in the $p$-plane, and the portion of the negative $z$-axis between the branch points, are drawn heavy. The letters (a), (b), . . , in the two drawings represent corresponding points, and the numbers written below them are their coordinates. Thus (a) is the point $z=\infty$, and transforms into $p=-\lambda s /(\lambda \pm 1)$. The singular points of the transformation are at (b) and (c).

* It will be noted that (16) is equivalent to an inversion in $p$, followed by (15); also that (17) is equivalent to a translation in $p$, followed by (15), and then by a translation in $z$. The set of transformations is closed under inversions in $z$. (11).

By setting $\lambda=1, \mu=\alpha, r=s=\infty$, and $\nu=0$ or $\alpha$ in (17), we get (10) and 
The second form is

$$
\begin{array}{ll}
z=\frac{r^{2}}{\lambda^{2}(s p+1)^{2}-1}, & s \geqq 0, \\
& \lambda \geqq 1,
\end{array}
$$

which represents the configuration shown in Fig. 8. In this case the boundary of the transformation consists of a part of the negative $p$-axis, together with the vertical line through $p=-1 / s$. The singular points of the transformation are at (b) and (c).

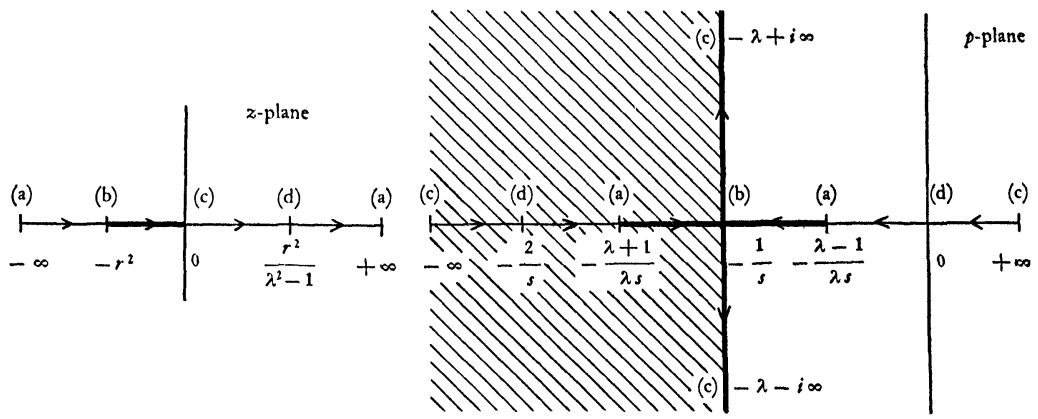

Fig. 8

The third form is

$$
\begin{array}{cl}
z+\nu=\frac{r^{2}(p+\mu)^{2}}{\lambda^{2}(p+\mu+s)^{2}-(p+\mu)^{2}}, & s \geqq 0, \\
0 \leqq \nu \leqq \frac{r^{2} \mu^{2}}{\lambda^{2}(s+\mu)^{2}-\mu^{2}}, &
\end{array}
$$

for which the configurations are shown in Fig. 9. They are similar, except for the positions of the origins, to Fig. 7. The singular points are again at (b) and (c).

Finally, we notice that, if we had transformed $F^{*}(p)$ into a function of $z$ by the use of (13), and if we had succeeded in obtaining a Stieltjes expansion for the latter, it would have been of the form (4), which becomes in terms of $p$

$$
f(z)=\frac{1}{a_{1} \frac{(a p+b)(\alpha p+\beta)}{(c p+d)(\gamma p+\delta)}+\frac{1}{a_{2}+\cdots}}
$$


This form in itself is not suitable for design purposes. But

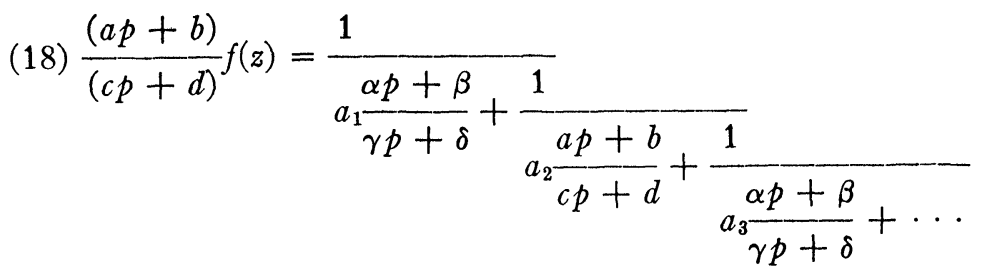

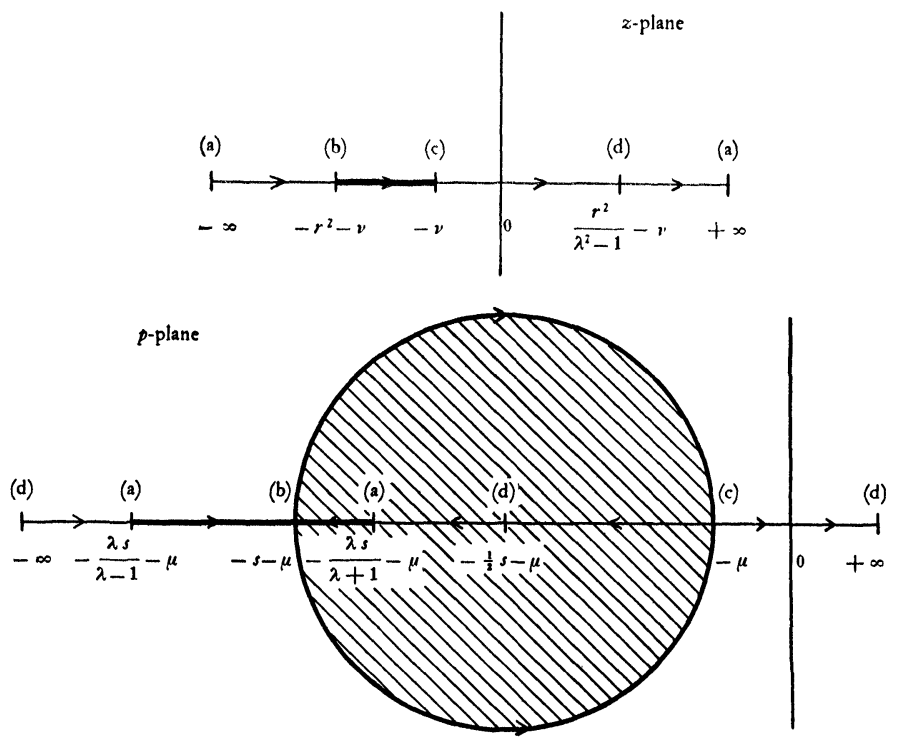

FIG. 9

is suitable for such uses. So also is

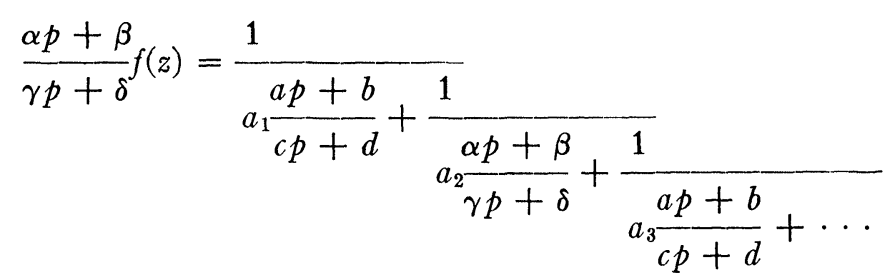

Hence, in using the transformation (13) for design purposes we would usually $\dagger$ identify $f^{*}(z)$ with the transformed of

$\dagger$ These are not the only possible bilinear multipliers which can be successfully used. However, we shall see that they serve our purpose remarkably well. 
either $(c p+d) F^{*}(p) /(a p+b)$ or $(\gamma p+\delta) F^{*}(p) /(\alpha p+\beta)$ in order that $F^{*}(p)$ might itself appear in one of the forms (18) or (19). The additional factors introduce no objectionable singularities into $f^{*}(z)$, since the vanishing of either $a p+b$, $c p+d, \alpha p+\beta$ or $\gamma p+\delta$ leads to either $z=0$ or $z=\infty$, both of which are "negative real values of $z$." Inspection shows, moreover, that unless these points are essential singularities, the one at $z=0$ must be a pole, and the one at $z=\infty$ a zero.

6. Theorems Relating to Redistribution of Singularities. Suppose, now, that we are given a function $F^{*}(p)$ whose singularities, together with those of its reciprocal, are all more than $1 / s$ units ( $s$ finite) to the left of the imaginary $p$-axis. Then by the use of the transformation (16), Fig. 8, we can obtain a function $f^{*}(z)$ with branch points at 0 and $-r^{2}$, and no other singularities or zeros in its one branch except possibly a simple zero at $\infty$. Moreover, the length $r^{2}$ of the cut in the z-plane may be finite.

If on the other hand we deal with Fig. 7 and suppose that either the given function $F^{*}(p)$ or its reciprocal is singular at $p=0$, but that all other singularities can be enclosed in the interior of a finite circle, it is a simple matter to show that all the essential facts of the last paragraph are again true. Hence we have the following theorem.

TheOREM 8. From any $F^{*}(p)$ all the singularities of which, together with those of its reciprocal, lie at least $1 / s$ units to the left of the imaginary p-axis, (or all the singularities of which, together with those of its reciprocal, except one at the origin, can be enclosed in the interior of a circle of finite radius), we can obtain a function $f^{*}(z)$ in at least two ways $\dagger$ which, together with its reciprocal, will be regular everywhere in the cut z-plane except at the two finite branch points 0 and $-r^{2}$, and except also for a simple zero at $\infty$. It has, moreover, at all points of the cut except the ends, a regular analytic continuation either from above or below.

$\dagger$ Corresponding to the two methods of identification discussed in $\$ 3$. 
We shall, in this paper, have little to say about functions $F^{*}(p)$ which do not satisfy the conditions of Theorem 8 , since they lead us into the ambiguities of Theorem 6 . However, we state the following theorem without proof:

THEOREM 9. If $F^{*}(p)$ and its reciprocal have no singular points to the right of the imaginary axis, but if the conditions of Theorem 8 can nevertheless not be met, the transformation (13) can still produce a function $f^{*}(z)$ the singularities of which, together with those of its reciprocal, are all located on the negative real axis. In this case, however, the entire negative z-axis is a cut, and the possibility of analytic continuation can in general not be asserted for every point on it.

If $F^{*}(p)$ or its reciprocal has a singular point to the right of the imaginary p-axis no transformation of the form (13) can carry it into an $f^{*}(z)$ the zeros and singularities of which all lie on the negative real axis.

We have, then, found a way of overcoming the second of our difficulties; for we have found a method of creating, from any function $F^{*}(p)$ whose singularities are all to the left of, or on, the imaginary axis, another function $f^{*}(z)$ whose singularities are located as required by the Stieltjes theory; and this has been accomplished in such a way, moreover, that the existence of an expansion of the form (4) for $f^{*}(z)$ implies the existence of a usable expansion of the form (18) or (19) for $F^{*}(p)$ also. We now turn our attention to the third difficulty: the possibility that $f^{*}(z)$ may not generate a $\Phi(x)$ of suitable character.

7. Existence Theorems concerning the Auxiliary Function $\Phi(x)$. To begin with, we introduce a definition which will aid us in avoiding much circumlocution .

The function $f(w)$ is said to be "admissibly" singular at the point $w=w_{0}$, if it possesses there a simple pole and/or $\dagger$ an essential singularity of such a character that $\ddagger$

$\dagger$ We do not wish to exclude such functions as $\left(w-w_{0}\right)^{1 / 2}+1 /\left(w-w_{0}\right)$.

$\ddagger$ It is obvious that this condition excludes isolated essential singularities. It includes many algebraic and logarithmic branch points, however. 


$$
\left|\left(w-w_{0}\right)^{k} f(w)\right|<M, \quad 0 \leqq\left|w-w_{0}\right|<\delta,
$$

where $\delta$ and $M$ are any positive numbers other than 0 and $\infty$, and where $0 \leqq k<1$.

We can then readily prove the following theorem.

TheORem 10. If the function $F^{*}(p)$ and its reciprocal have none but admissible singularities on the imaginary $p$-axis, the $f^{*}(z)$ obtained either from $[(c p+d) /(a p+b)] F^{*}(p)$ or from $[\gamma p+\delta) /(\alpha p+\beta)] F^{*}(p)$ by the use of (13) will have only admissible singularities on the negative real axis. The same is true of $1 / f^{*}(z)$.

At all but the branch points of the transformation this is immediately obvious, for the character of a singularity is never altered by a conformal transformation. At the singular points the transformation substantially replaces $p-p_{0}$ by $\left(z-z_{0}\right)^{1 / 2}$; whence even if the additional bilinear terms in $p$ happen to be singular at such a point they cannot render $f^{*}(z)$ inadmissibly singular. We need hardly fill in the details of the proof.

Next we note that, since $F^{*}(p)$ is real for every real positive $p, f^{*}(z)$ is also real for every real positive $z$. This follows from a very simple study of analytic continuation in the p-planes of Figs. 7, 8 and 9. Then from an equally easy study of analytic continuation in the z-planes we find that $f^{*}(x+i y)$ and $f^{*}(x-i y)$ are conjugate complex. Hence, if we introduce the integral

$$
G(z)=\int_{\alpha}^{z} f^{*}(z) d z,
$$

the integration being performed along any path which passes from the (arbitrary) positive real $\alpha$ to $z$ without touching the negative real axis, we see at once that $G(x+i y)$ and $G(x-i y)$ are conjugate complex. But by (8)

$$
\Phi(x)=\lim _{y \rightarrow 0} \frac{G(-x+i y)-G(-x-i y)}{2 \pi i} .
$$

Hence if this limit exists at all it is real. 
As for the existence of the limit, if $f^{*}(z)$ is regular and capable of analytic continuation across the axis, or if it possesses a singularity of the sort (20), $G(-x+i y)$ and $G(-x-i y)$ approach well-defined finite limits. If on the other hand $f^{*}(z)$ has a simple pole at $z=-x$, it may be written as $\alpha /(z+x)+f_{1}^{*}(z)$, where $f_{1}^{*}(z)$ has the same analytical character as $f^{*}(z)$ at all other points than $-x$, and at $-x$ satisfies (20). Then by actual integration

$$
\Phi(x)=\frac{1}{2} \alpha+\lim _{y \rightarrow 0} \frac{G_{1}(-x+i y)-G_{1}(-x-i y)}{2 \pi i},
$$

where $G_{1}(z)$, being derived from $f_{1}(z)$, is known to have welldefined limits. Hence we have the following theorem.

THEOREM 11. Any function $f^{*}(z)$ which is real for real positive $z$, regular for all but real negative $z$, and only admissibly singular for real negative z, generates a well-defined real function $\Phi(x)$.

We are now prepared to state, as a consequence of Theorems 8, 9, 10 and 11, the following theorem, which has been the objective of the present section.

THEOREM 12. From any function $F^{*}(p)$ which is real for positive real values of $p$, which has no singularities to the right of the imaginary axis, and none but admissible singularities on the imaginary axis, at least two functions $f^{*}(z)$ can be produced by the use of the transformation (13), each of which will generate a $\Phi(x)$ which is weli-defined for every $x$.

If, in addition, the conditions of Theorem 8 are met by $F^{*}(p), \Phi(x)$ will be of limited variation, and constant for $x>r^{2}$.

Only the last paragraph of the theorem requires proof. That $\Phi(x)$ is constant for $x>r^{2}$ follows at once from (8) and from the fact, stated in Theorem 8 , that $f^{*}(z)$ is regular for $|z|>r^{2}$.

To prove that $\Phi(x)$ is of limited variation we exclude for a moment the possibility of poles at the branch points, con- 
sidering only singularities of the type (20). Then, since $f^{*}(z)$, and consequently $G(z)$ also, is regular as we approach the negative real axis from above and below, we have

$$
\begin{aligned}
\frac{d \Phi}{d x} & =\lim _{y \rightarrow 0} \frac{1}{2 \pi i} \frac{d}{d x}[G(-x+i y)-G(-x-i y)] \\
& =\frac{1}{2 \pi i} \lim _{y \rightarrow 0}\left[f^{*}(-x-i y)-f^{*}(-x+i y)\right]
\end{aligned}
$$

for all values of $x$ except $x=0$ and $x=r^{2}$. Hence the variation of $\Phi(x)$ is

$$
\operatorname{Var} \Phi=\int_{0}^{r^{2}}\left|\frac{d \Phi}{d x}\right| d x \leqq \frac{1}{\pi} \int_{0}^{r^{2}}\left|f^{*}(-x)\right| d x .
$$

We now consider the half of this interval nearest the origin. We know that, over the range $0<x \leqq \delta,(20)$ is satisfied. We also know that $\left|f^{*}(z)\right|$ is bounded in the interval $\delta \leqq x \leqq r^{2} / 2$. Call its upper bound $m$. Then if we take $M_{1}$ as the larger of the two quantities $M$ and $\left(2 / r^{2}\right)^{k} m$, it will be true that

$$
\left|f^{*}(-x)\right| \leqq M_{1} / x^{k}
$$

over the entire interval $0<x \leqq r^{2} / 2$. A similar statement (with $M=M_{2}$ ) can be made for the other half of the interval, $r^{2} / 2 \leqq x<r^{2}$. Hence

$$
\operatorname{Var} \Phi \leqq\left(M_{1}+M_{2}\right)\left(r^{2} / 2\right)^{1-k} /(1-k),
$$

which proves our theorem, provided the singularities of $f^{*}(z)$ are of the type (20).

If, on the other hand, $f^{*}(z)$ possesses a pole at either $z=0$ or $z=-r^{2}$, or both, these poles will have no other effect then to introduce finite discontinuities into $\Phi(x)$ equal to their residues; and it is seen at once that the theorem will still be true.

Let us now take stock of our study from the standpoint of network design. We know that, from any $F^{*}(p)$ which satisfies the conditions of Theorem 8 , we can obtain an $f^{*}(z)$ the singularities of which are on the negative real $z$-axis, and 
which generates a $\Phi(x)$ the variation of which is bounded, and which is constant for $x>r^{2}$. We may, therefore, replace $\Phi(x)$ by the difference of two functions, $\Phi_{1}(x)$ and $\Phi_{2}(x)$, both of which are monotonic, non-decreasing, and both finite for $x>r^{2}$. Hence, by Theorem 5, the functions $f_{1}(z)$ and $f_{2}(z)$ which correspond to $\Phi_{1}(x)$ and $\Phi_{2}(x)$ possess Stieltjes fractions. That is, when the conditions of Theorem 8 are satisfied, $f^{*}(z)$ generates a $\Phi(x)=\Phi_{1}(x)-\Phi_{2}(x)$, to which corresponds an $f(z)=f_{1}(z)-f_{2}(z)$, which can be written as the difference of two Stieltjes fractions.

As it stands, this result has no practical utility, for we cannot connect two networks in such a way that their combined impedance is the difference of their separate impedances. If, however, we can find a way of obtaining a fraction with positive coefficients the $\Phi(x)$ corresponding to which is monotonic non-increasing, [say a fraction the limit of which is $\left.f_{2}^{\prime}(z)\right]$, then $f^{\prime}(z)=f_{1}(z)+f_{2}^{\prime}(z)$ will also generate the same $\Phi(x)$ as $f^{*}(z)$, and may turn out to be identical with it. Such a result would be usable. This leads us, then, to the search for a form of fraction the $\Phi(x)$ of which is monotonic non-increasing.

8. Functions for which $\Phi(x)$ is Monotonic Decreasing. We can easily show that if $f^{*}(z)$ gives rise to a monotonic non-increasing $\Phi(x)$, certain related functions give rise to monotonic non-decreasing $\Phi$ 's. Chief among these are $z f^{*}(z), 1 / f^{*}(z)$ and $f^{*}(1 / z)$.

We treat first the case of $z f^{*}(z)$. Wherever $f^{*}(z)$ is regular as it approaches the negative real axis from above and below, $f_{1}(z)=z f^{*}(z)$ is also, and therefore either $f^{*}(z)$ or $f_{1}^{*}(z)$ can be substituted in (23). Thus we obtain

$$
\begin{aligned}
\frac{d \Phi_{1}(x)}{d x}= & \frac{1}{2 \pi i} \lim _{y \rightarrow 0}\left[f_{1}^{*}(-x-i y)-f_{1}^{*}(-x+i y)\right] \\
= & \frac{-x}{2 \pi i} \lim _{y \rightarrow 0}\left[f^{*}(-x-i y)-f^{*}(-x+i y)\right] \\
& -\frac{1}{2 \pi} \lim _{y \rightarrow 0} y\left[f^{*}(-x-i y)+f^{*}(-x+i y)\right] .
\end{aligned}
$$


The last limit, however, vanishes owing to the finiteness of $*(-x \pm i y)$. Hence by (23) we have

$$
\frac{d \Phi_{1}(x)}{d x}=-x \frac{d \Phi}{d x}
$$

or

$$
d \Phi_{1}(x)=-x d \Phi(x) .
$$

On the other hand, at a pole the residue of $f_{1}^{*}(z)$ is $-x$ times the residue of $f^{*}(z)$. That is, (24) is still true.

The extension to singularities of the type (20) is almost equally simple. It consists merely in showing that at such a point both $\Phi(x+\delta x)-\Phi(x-\delta x)$ and $\Phi_{1}(x+\delta x)-\Phi_{1}(x-\delta x)$ vanish with $\delta x$. There is, then, no discontinuity at the point, and (24) rules at every neighboring point. Hence we may state the following theorem.

THEOREM 13. If the singularities of $f^{*}(z)$ are all on the negative real axis and are of admissible type, and if it generates a monotonic function $\Phi(x)$, then the function $f_{1}^{*}(z)=z f^{*}(z)$ generates a function $\Phi_{1}(x)$ which is monotonic in the opposite sense. More specifically, the two are related by equation (24).

We could frame somewhat similar proofs to show that the $\Phi^{\prime}$ 's generated by $f^{*}(1 / z)$ and $1 / f^{*}(z)$ are monotonic in the opposite sense to that generated by $f^{*}(z)$ itself. However we have no occasion to use them in the present paper.

The significance of Theorem 13 for our purposes lies in the fact that, if $f^{*}(z)$ possesses a Stieltjes expansion of the form $(4), f_{1}^{*}(z)=z f^{*}(z)$ will have the expansion

$$
f_{1}^{*}(z)=\frac{1}{a_{1}+\frac{1}{a_{2} z+\frac{1}{a_{3}+\cdots}}}
$$

This form of expansion, therefore, is appropriate for functions which generate monotonic decreasing $\Phi$ 's. Moreover, in view of the remarks made at the end of $\$ 7$, if we were given a 
function of bounded variation, we would naturally seek to expand it in the sum of two fractions, one of form (4) and one of form (25).

9. The Difference Function, $\delta(z)$. We now have methods for overcoming, to a large degree, our second and third difficulties. But while we are thus enabled to effect an expansion of

$$
f(z)=\int_{0}^{b} \frac{d \Phi(x)}{z+x}
$$

in a usable form, we still do not know that this $f(z)$ and $f^{*}(z)$ are identical. We must now study this fourth difficulty.

If we write

$$
\delta(z)=f^{*}(z)-f(z)
$$

we know that

$$
\frac{1}{2 \pi i} \int \delta(z) d z=0,
$$

the integral being taken about any (every) circle of radius $x$ about the origin. We ask what sort of function $\delta(z)$ can be. We see at once that

(a) It is regular except, perhaps, at negative real values of $z$. This follows from the fact that it is the difference of two regular functions.

(b) It may be an entire function if $f^{*}(z)$ is singular at infinity; or if $f^{*}(z)$ is regular at infinity $\delta(z)$ may be a constant. That any entire function satisfies the requirement (26) is obvious. However, if $f^{*}(z)$ is regular at infinity, $\Phi(x)$ is constant for $x$ greater than some finite value $r^{2} ; f(z)$ is therefore also regular for $|z|>r^{2}$. Hence the same will be true of $\delta(x)$ as well.

(c) It may have any number of isolated singular points the contour integrals about which are zero. Such singularities, however, can only occur where $f^{*}(z)$ is itself singular. For example,

† Perron. loc. cit., p. 369. 


$$
\delta(x)=\frac{1}{(z+x)^{2}},
$$

which has residue zero, is possible. To establish that $\delta(z)$ is regular wherever $f^{*}(z)$ is regular, we need only show that this is true of $f(z)$. Let us suppose, then, that $f^{*}(z)$ is represented on a Riemann surface of any number of sheets, connected along certain segments of the negative real axis, and that it has no singularities in the interval $\left(-x_{2},-x_{1}\right)$. Elsewhere on the negative real axis it may be of any analytic character whatever consistent with the fact that it generates $\Phi(x)$. Then the $G(z)$ defined by (21) is regular for every $z$ not on the negative real axis, and also for values of $z$ between $\left(-x_{2},-x_{1}\right)$; though in the case of this interval it may take different values $F_{1}(z)$ and $F_{2}(z)$ as we approach the axis from above and below. It follows, then, that

$$
\Phi(z)=\frac{F_{1}(-z)-F_{2}(-z)}{2 \pi i}
$$

is a regular analytic function of $z$ in the vicinity of $\left(x_{1}, x_{2}\right)$. Along the real axis this function is, by definition, identical with $\Phi(x)$. Then by a well known theorem $\dagger$, it is also true that

$$
\int_{x_{1}}^{x_{2}} \frac{d \Phi(x)}{z+x}=\int_{x_{1}}^{x_{2}} \frac{d \Phi}{d x} \frac{d x}{z+x}
$$

is regular-though perhaps multiple valued-between $-x_{2}$ and $-x_{1}$. On the other hand

and

$$
\int_{0}^{x_{1}} \frac{d \Phi(x)}{z+x}
$$

$$
\int_{x_{2}}^{\infty} \frac{d \Phi(x)}{z+x}
$$

are both analytic in this same region. $\ddagger$ Since $f(z)$ is the sum of these three integrals, it too must be regular in the interval $\left(x_{1}, x_{2}\right)$.

$\dagger$ Goursat, Cours d'Analyse, vol. 2, p. 254.

$\ddagger$ Perron, loc. cit., p. 369 . 
(d) $\delta(z)$ does not admit of non-essential cuts. For suppose $\left(-x_{2},-x_{1}\right)$ were such a cut: then it would be possible to effect analytic continuation not only of $\delta(z)$, but of the integral

$$
\Delta(z)=\int_{\alpha}^{z} \delta(z) d z
$$

also, across this boundary. Let us call the value taken by $\Delta(z)$ as reached through the upper half of the plane $\Delta_{1}(z)$, and that reached through the lower half $\Delta_{2}(z)$. Then $\Delta_{1}-\Delta_{2}$ possesses a well-defined set of derivatives, which we can obtain by choosing $d z$ in any manner we like. For example, we may choose $d z=d x$ if we wish. But by (26) $\Delta_{1}-\Delta_{2}$ is identically zero along the negative real axis; hence all the derivatives vanish. That is $\Delta_{1}(z)=\Delta_{2}(z)$, and the function $\delta(z)$ is its own analytic continuation across $\left(-x_{2},-x_{1}\right)$.

As a final result of all these considerations we may enunciate the following theorem.

THEOREM 14. Two functions $f_{1}(z)$ and $f(z)$ which are regular everywhere except on the negative real axis and generate the same $\Phi(x)$ can differ at most by a function $\delta(z)$ which is single-valued and regular, except perhaps at certain points where one or both of the functions $f_{1}$ and $f$ are singular. $\dagger$ At such points $\delta(z)$ may possess isolated singularities of residue zero; or, in case the singularities of $f_{1}$ or $f$ are everywhere dense in an interval $\left(-x_{2},-x_{1}\right)$, the same may be true of $\delta$ also; but in this latter case the relation (26) will still be satisfied.

A special case of peculiar interest is the type of function derived through the use of the transformation (13), when $F^{*}(p)$ satisfies the conditions of Theorem 8 . In such cases, $f^{*}(z)$ has singularities at only $-y^{2}$ and 0 ; hence $\delta(z)$ can have singularities nowhere else. Usually it is found to be a constant.

$\dagger$ Our proof has assumed one function to correspond to $\Phi(x)$. But the difference between any two functions which generate the same $\Phi(x)$ is the sum of two $\delta$ 's of the type with which our proof deals, whence the theorem is true as stated. 
10. An Example. As an example of this process of expansion, let us consider

$$
f^{*}(z)=\left(z+\frac{1}{2}\right)\left(z^{2}+z\right)^{1 / 2}-z^{2}-z,
$$

a function which is everywhere regular (including $\infty$ ) except at the winding points $z=0$ and $z=-1$. We wish to represent that branch of it for which the radical is positive for positive real $z$. We readily find that it generates

$$
\begin{cases}\Phi(x)=-\frac{1}{3 \pi}\left(x-x^{2}\right)^{3 / 2}, & 0<x<1, \\ \Phi(x)=0, & 1<x ;\end{cases}
$$

or, since it is the derivative ${ }^{\dagger}$ of $\Phi$ rather than $\Phi$ itself which is of interest,

$$
\frac{d \Phi}{d x}=\frac{x}{\pi}\left(x-x^{2}\right)^{1 / 2}-\frac{1}{2 \pi}\left(x-x^{2}\right)^{1 / 2} .
$$

We can, then, divide $\Phi$ into a monotonic non-decreasing term $\Phi^{\prime}$ and a monotonic non-increasing term $\Phi_{1}^{\prime \prime}$ by choosing

and

$$
\frac{d \Phi^{\prime}}{d x}=\frac{x}{\pi}\left(x-x^{2}\right)^{1 / 2}
$$

$$
\frac{d \Phi_{1}^{\prime \prime}}{d x}=-\frac{1}{2 \pi}\left(x-x^{2}\right)^{1 / 2} .
$$

The function $f^{\prime}(z)$ which corresponds to $\Phi^{\prime}$ is found directly from (5) to be

$$
\begin{aligned}
f^{\prime}(z) & =\int_{0}^{1} \frac{d \Phi^{\prime}}{d x} \frac{d x}{z+x} \\
& =z\left(z^{2}+z\right)^{1 / 2}-z^{2}-\frac{z}{2}+\frac{1}{8} .
\end{aligned}
$$

We know, then, that this function possesses a Stieltjes expansion.

$\dagger$ Since $\Phi(x)$ is differentiable, the Stieltjes integral $\int d \Phi /(z+x)$ reduces simply to $\int(d \Phi / d x) d x /(z+x)$. 
To find the residual function $f_{1}^{\prime \prime}(z)$ which corresponds to $\Phi_{1}^{\prime \prime}$, we adopt the process suggested by Theorem 13 and compute the auxiliary function

$$
f^{\prime \prime}(z)=\frac{f_{1}^{\prime \prime}(z)}{z}
$$

which corresponds to

$$
\frac{d \Phi^{\prime \prime}}{d x}=-\frac{1}{x} \frac{d \Phi_{1}^{\prime \prime}}{d x}=\frac{1}{2 \pi} \frac{\left(x^{2}-x\right)^{1 / 2}}{x} .
$$

Again, we find from (5) that

$$
f^{\prime \prime}(z)=\frac{1}{2}\left(\frac{\left(z^{2}+z\right)^{1 / 2}}{z}-1\right) .
$$

This also possesses a Stieltjes expansion. Then

$$
f_{1}^{\prime \prime}(z)=\frac{1}{2}\left[\left(z^{2}+z\right)^{1 / 2}-z\right]
$$

possesses an expansion of the type

$$
f^{\prime \prime}(z)=\frac{1}{a_{1}+\frac{1}{a_{2} z+\cdots}} .
$$

By adding together (29) and (30) we get the function which corresponds to (28). It is

$$
f(z)=\left(z+\frac{1}{2}\right)\left(z^{2}+z\right)^{1 / 2}-z^{2}-z+\frac{1}{8} .
$$

Now, by comparing this with (27), we find that the two differ by only a constant, which is what we would expect in the light of Theorem 14.

We could also have proceeded as follows: Since $\Phi(x)$, as given by (28), decreases steadily up to $x=1 / 2$, when it reaches the value $-1 /(24 \pi)$, and then increases, we may choose 
1929.]

$$
\left\{\begin{array}{lr}
\Phi^{\prime}=0, & 0<x<\frac{1}{2}, \\
\Phi^{\prime}=\frac{1}{24 \pi}-\frac{1}{3 \pi}\left(x-x^{2}\right)^{3 / 2}, & \frac{1}{2}<x<1, \\
\Phi^{\prime}=\frac{1}{24 \pi}, & 1<x ; \\
\Phi_{1}^{\prime \prime}=-\frac{1}{3 \pi}\left(x-x^{2}\right)^{3 / 2}, & 0<x<\frac{1}{2}, \\
\Phi_{1}^{\prime \prime}=-\frac{1}{24 \pi}, & \frac{1}{2}<x .
\end{array}\right.
$$

In other words,

and

$$
\frac{d \Phi^{\prime}}{d x}=\frac{1}{\pi}\left(x-\frac{1}{2}\right)\left(x-x^{2}\right)^{1 / 2}, \quad \frac{1}{2}<x<1,
$$

$$
\frac{d \Phi_{1}^{\prime \prime}}{d x}=-\frac{1}{\pi}\left(\frac{1}{2}-x\right)\left(x-x^{2}\right)^{1 / 2}, \quad 0<x<\frac{1}{2},
$$

the values of the derivatives being zero outside the indicated ranges. Then by direct computation we find for $f^{\prime}(z)$

$$
\begin{aligned}
f^{\prime}(z)= & \frac{1}{4 \pi(2 z+1)}-\frac{1}{16}-\frac{z^{2}+z}{2}+\frac{2 z+1}{4}\left(z^{2}+z\right)^{1 / 2} \\
& -\frac{1}{\pi} \frac{z^{2}+z}{2 z+1}\left[F\left(1,1, \frac{1}{2} ; \frac{1}{(2 z+1)^{2}}\right)-1\right],
\end{aligned}
$$

where $F(\alpha, \beta, \gamma ; x)$ stands for the hypergeometric function

$$
F(\alpha, \beta, \gamma ; x)=1+\frac{\alpha \beta}{1 \gamma} x+\frac{\alpha \cdot \alpha+1 \cdot \beta \cdot \beta+1}{1 \cdot 2 \cdot \gamma \cdot \gamma+1} x^{2}+\cdots .
$$

Similarly, we set $d \Phi^{\prime \prime} / d x=-(1 / x)\left(d \Phi_{1}^{\prime \prime} / d x\right)$, compute $f^{\prime \prime}(z)$, and then make $f_{1}^{\prime \prime}(z)=z f^{\prime \prime}(z)$. The result is

$$
\begin{aligned}
f_{1}^{\prime \prime}(z)=\frac{z}{2 \pi(2 z+1)} & -\frac{z^{2}+z}{2}+\frac{2 z+1}{4}\left(z^{2}+z\right)^{1 / 2} \\
& +\frac{1}{\pi} \frac{z^{2}+z}{2 z+1}\left[F\left(1,1, \frac{1}{2} ; \frac{1}{(2 z+1)^{2}}\right)-1\right] .
\end{aligned}
$$


Adding $f^{\prime}$ and $f_{1}^{\prime \prime}$ together we get

(33) $f(z)=\left(\frac{1}{4 \pi}-\frac{1}{16}\right)-z^{2}-z+\left(z+\frac{1}{2}\right)\left(z^{2}+z\right)^{1 / 2}$.

This result again differs from (27) only to the extent of an additive constant. This time, however, the constant is smaller than before, being 0.017 instead of 0.125 .

This value $(4-\pi) /(16 \pi)$ is, in fact, the smallest difference which can exist between the sum of two fractions of the form

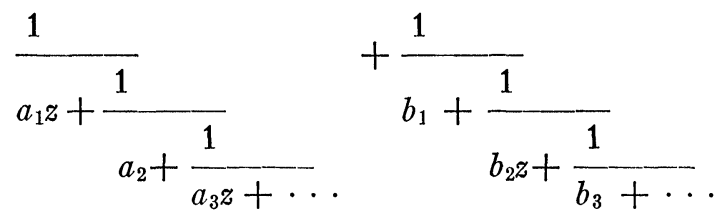

and the function (27), as we can readily show by the following argument.

It is evident that, at $z=\infty$, such a pair of fractions takes the value $1 / b_{1}$. However, it is known (Theorem 2) that $1 / b_{1}$ is equal to $\Phi^{\prime \prime}(\infty)$. Hence the fraction will have its least value at $\infty$ when $\Phi^{\prime \prime}(\infty)$ is smallest. We see at once from the relation between $\Phi^{\prime \prime}$ and $\Phi_{1}^{\prime \prime}$ that $\Phi^{\prime \prime}(\infty)$ will be smallest when $\Phi_{1}^{\prime \prime}(x)$ is given for every $x$ the smallest value which is consistent with the requirement that $\Phi^{\prime}$ shall nowhere decrease. This, however, is obviously the function $\Phi_{1}^{\prime \prime}(x)$ which we have adopted in (32). From it we find by direct computation that $\Phi^{\prime \prime}(\infty)=(4+\pi) /(16 \pi)$. This, then, is the smallest value which $f(\infty)$ can have. We easily see from (27), however, that $f^{*}(\infty)=1 / 8$. Hence the smallest difference that can exist between the two is $(4-\pi) /(16 \pi)$, which is, indeed, the constant term in (33).

11. The Final Process for Obtaining a Usable Expansion. In the discussions of $\$ \$ 7-10$ we have tacitly assumed that an expansion of the form (34) is satisfactory for purposes of design; and so it is if $z$ is synonymous with our frequency variable $p$, for as we have seen in $\$ 1$ the two fractions then correspond to constructible networks. But when the func- 
tion $f^{*}(z)$ has been identified with $(c p+d) F^{*}(p) /(a p+b)$ or with $(\gamma p+\delta) F^{*}(p) /(\alpha p+\beta)$ by use of the transformation (13), the situation is not quite so simple, and something still remains to be accomplished.

To see why this is true, let us abbreviate the expressions $(a p+b) /(c p+d)$ and $(\alpha p+\beta) /(\gamma p+\delta)$ to $q_{1}$ and $q_{2}$, so that $z=q_{1} q_{2}$. Then from (34) we may get a pair of fractions representing $F(p)=q_{1} f(z)$. The terms of one of these are of the form $a q_{1}$ and $a q_{2}$ and are therefore constructible. But in the other, terms of the form $b q_{1}{ }^{2} q_{2}$ appear, and these cannot be interpreted in electrical terms. $\dagger$ Hence the result, in exactly this form, is useless.

This difficulty can be overcome by separating $F^{*}(p)$ itself into two components each of which generates an expansion of usable form, instead of dealing with the transformed function $f^{*}(z)$.

In order to make our study somewhat more readable, we shall think entirely in terms of the transformation (15) and its accompanying Fig. 7. This is indeed quite general, for all distributions of singularities which can be enclosed by the boundary of (17) can also be enclosed by (15), and, as we have already observed, (16) can be obtained from (15) by a simple inversion on $p$. Then we have

$$
q_{1}=\frac{r p}{(\lambda+1) p+s}, \quad q_{2}=\frac{r p}{(\lambda-1) p+s} .
$$

We shall also assume $F^{*}(p)$ to be broken up into the sum of two components $G^{*}(p)$ and $H^{*}(p)$ by a procedure which will become clear as we proceed, and shall indicate the three functions of $z$ into which they transform by $f^{*}(z), g^{*}(z)$ and $h^{*}(z)$. That is, $f^{*}(z) \equiv F^{*}(p), \cdots$.

We shall be interested in the values of all these various functions along the upper and lower edges of the cut in the

$\dagger$ We assume that $f^{*}(z)$ has been identified with $F^{*}(p) / q_{1}$. If it has been identified with $F^{*}(p) / q_{2}$ the fractions are slightly different, but still unsatisfactory. 
$z$-plane. In the case of $q_{1}$ and $q_{2}$ we readily find them to be

$$
\left\{\begin{array}{l}
r q_{1}=x \pm i\left(r^{2} x-x^{2}\right)^{1 / 2} \\
r q_{2}=-x \pm i\left(r^{2} x-x^{2}\right)^{1 / 2}
\end{array}\right.
$$

where the upper and lower signs apply to the upper and lower borders of the cut, respectively. About the values of $f^{*}$, $g^{*}$ and $h^{*}$ we can say nothing definite, but we introduce the notation

$$
\left\{\begin{array}{l}
f^{*}(-x)=u_{f} \pm i v_{f} \\
g^{*}(-x)=u_{g} \pm i v_{g} \\
h^{*}(-x)=u_{h} \pm i v_{h}
\end{array}\right.
$$

Then in terms of this notation, we readily find from (23) that the $\Phi$ 's generated by $F^{*}(p), G^{*}(p) / q_{2}$ and $H^{*}(p) / q_{1}$, are, respectively

$$
\left\{\begin{aligned}
\pi \frac{d \Phi_{f}(x)}{d x} & =-v_{f}, \\
r \pi \frac{d \Phi_{g}(x)}{d x} & =u_{g}\left(\frac{r^{2}}{x}-1\right)^{1 / 2}+v_{g}, \\
r \pi \frac{d \Phi_{h}(x)}{d x} & =u_{h}\left(\frac{r^{2}}{x}-1\right)^{1 / 2}-v_{h} .
\end{aligned}\right.
$$

Now, what we purpose to show is, that so long as $F^{*}(p)$ is bounded along the entire boundary of the transformation, $\dagger$ $G^{*}(p)$ and $H^{*}(p)$ can always be so chosen that the left hand members of the second and third of equations (38) are everywhere positive. $\Phi_{g}(x)$ and $\Phi_{h}(x)$ then satisfy the conditions of Theorem 5. From them we can therefore obtain convergent Stieltjes fractions in the variable $z=q_{1} q_{2}$; whence, calling these fractions $G(p) / q_{2}$ and $H(p) / q_{1}$, respectively, we have

$\dagger$ This condition is sufficient, but not necessary, as we shall see in $\S 12$. 


$$
\left\{\begin{array}{rl}
G(p)= & \frac{1}{a_{1} q_{1}+\frac{1}{a_{2} q_{2}+\frac{1}{a_{3} q_{1}+\cdots}}} \\
H(p)= & \frac{1}{b_{1} q_{2}+\frac{1}{b_{2} q_{1}+\frac{1}{b_{3} q_{2}}+\cdots}}
\end{array},\right.
$$

both of which are constructible.

To show that this is so, we start with the first of equations (38) and set $v_{g}^{\prime}=v_{f}$ wherever $v_{f}$ is positive, and $v_{g}^{\prime}=0$ elsewhere. Similarly we put $v_{h}^{\prime}=v_{f}$ wher ever $v_{f}$ is negative, and zero elsewhere. $\dagger$ Thus we have

$$
v_{g}^{\prime}+v_{h}^{\prime}=v_{f} .
$$

Next we define two new functions of $z$ by the formulas

$$
\left\{\begin{array}{l}
g^{\prime}(z)=-\frac{1}{\pi} \int_{0}^{r^{2}} \frac{v_{g}^{\prime} d x}{z+x} \\
h^{\prime}(z)=-\frac{1}{\pi} \int_{0}^{r^{2}} \frac{v_{h}^{\prime} d x}{z+x} .
\end{array}\right.
$$

From (5), (40) and the first of equations (38) we see that $f^{\prime}(z)=g^{\prime}(z)+h^{\prime}(z)$ is either identical with $f^{*}(z)$, or else differs from it only to the extent of a function $\delta(z)$ the $\Phi$ of which is everywhere zero. Further, by an argument essentially similar to that in $\$ 10$ we may readily show that $g^{\prime}(z)$ and $h^{\prime}(z)$ are regular analytic functions as we approach the negative real axis from above and below, except perhaps at 0 and $-r^{2}$, and are bounded at these points. By analogy with (37) we shall call their values along the cut

$$
\left\{\begin{array}{l}
g^{\prime}(-x)=u_{g}^{\prime} \pm i v_{g}^{\prime}, \\
h^{\prime}(-x)=u_{h}^{\prime} \pm i v_{h}^{\prime}
\end{array}\right.
$$

$\dagger$ Any other method of division for which $v_{g}^{\prime}$ is everywhere positive and $v_{h}^{\prime}$ everywhere negative is satisfactory. 
the $v_{g}^{\prime}$ being by definition everywhere positive, the $v_{h}^{\prime}$ everywhere negative, while $u_{g}^{\prime}$ and $u_{h}^{\prime}$ are only known to be bounded.

Finally, we take the arbitrary step of defining $g^{*}(z)$ and $h^{*}(z)$ - and therefore also the $G^{*}(p)$ and $H^{*}(p)$ into which $F^{*}(p)$ is divided-by the equations

$$
\begin{aligned}
& g^{*}(z)=g^{\prime}(z)-M\left(\frac{r^{2}}{z}+1\right)^{1 / 2} \\
& h^{*}(z)=h^{\prime}(z)+M\left(\frac{r^{2}}{z}+1\right)^{1 / 2}+\delta(z),
\end{aligned}
$$

$M$ being a positive constant the value of which will be assigned later. Then we see that along the cut

$$
\begin{aligned}
& g^{*}(-x)=u_{g}^{\prime} \pm i v_{g}^{\prime} \pm i M\left(\frac{r^{2}}{x}-1\right)^{1 / 2} \\
& h^{*}(-x)=u_{h}^{\prime} \pm i v_{h}^{\prime} \pm i M\left(\frac{r^{2}}{x}-1\right)^{1 / 2}
\end{aligned}
$$

and upon comparing these values with (37) we can identify $u_{o}, v_{g}, u_{h}$ and $v_{h}$. Substituting the values thus found in the second and third of equations (38), we arrive at

$$
\left\{\begin{array}{l}
r \pi \frac{d \Phi_{g}(x)}{d x}=\left(u_{o}^{\prime}+M\right)\left(\frac{r^{2}}{x}-1\right)^{1 / 2}+v_{g}^{\prime}, \\
r \pi \frac{d \Phi_{h}(x)}{d x}=\left(u_{h}^{\prime}+M\right)\left(\frac{r^{2}}{x}-1\right)^{1 / 2}-v_{h}^{\prime} .
\end{array}\right.
$$

Now, since $u_{o}^{\prime}$ and $u_{h}^{\prime}$ are bounded, $M$ may be taken so large that the first terms are positive, while the terms $v_{g}^{\prime}$ and $-v_{h}^{\prime}$ are positive by definition. Hence both $\Phi_{g}(x)$ and $\Phi_{h}(x)$ are monotonic increasing and lead to Stieltjes fractions in $z$.

Thus we are assured that expansions of the form (39) really exist, where $G(p)$ and $H(p)$ are related to $F^{*}(p)$ by the equation

$$
F^{*}(p)=G(p)+H(p)+\Delta(p)
$$


$\Delta(p)$ being some function which generates a $\Phi$ that is everywhere zero. Hence we may state the following theorem.

ThEOREM 15. If $F^{*}(p)$ satisfies the conditions of Theorem 8 and is finite at the origin, it can be expressed as the sum of three functions $G(p), H(p)$ and $\Delta(p)$, the first two of which possess constructible expansions of the form (39), while the third generates a $\Phi$ which is everywhere zero.

We have thus avoided the difficulty to which attention was called at the beginning of the section.

12. An Example. We can make the material of $\$ 11$ somewhat more intelligible by means of an illustration.

Let us consider the function $\dagger$

$$
F^{*}(p)=\left(p^{2}+p+\frac{1}{2}\right) \log \frac{p+1}{p} .
$$

By using (15) in the form

$$
z=\frac{p^{2}}{(p+1)^{2}-p^{2}}
$$

(which means, of course, that $r=s=\lambda=1$ ), we readily convert $F^{*}(p)$ into

$$
f^{*}(z)=\left(z+\frac{1}{2}\right)\left(z+\frac{1}{2}+\left(z^{2}+z\right)^{1 / 2}\right) \log \frac{z+1}{z} .
$$

Along the negative real axis this takes the value

$$
\begin{aligned}
f^{*}(-x) & =\left(x-\frac{1}{2}\right)^{2} \log \frac{1-x}{x}+\pi\left(\frac{1}{2}-x\right)\left(x-x^{2}\right)^{1 / 2} \\
\pm & \left.i\left(\frac{1}{2}-x\right)\left(x-x^{2}\right)^{1 / 2} \log \frac{1-x}{x}-\pi\left(x-\frac{1}{2}\right)^{2}\right] \\
& \equiv u_{f} \pm i v_{f} .
\end{aligned}
$$

$\dagger$ This function has a pole at $\infty$, and is not bounded at the points 0 and -1 . Thus it violates the conditions laid down in Theorem 15. The choice of such a function is deliberate, as it serves to emphasize that the conditons, while sufficient, are not always necessary. 
This is the analog of the first of equations (37).

We now define

$$
\left\{\begin{array}{l}
v_{g}^{\prime}=\left(\frac{1}{2}-x\right)\left(x-x^{2}\right)^{1 / 2} \log \frac{1-x}{x}, \\
v_{h}^{\prime}=-\pi\left(x-\frac{1}{2}\right)^{2}
\end{array}\right.
$$

this definition serving the purpose of making $v_{0}^{\prime}$ everywhere positive and $v_{h}^{\prime}$ everywhere negative. Then we find from (41)

$$
\begin{aligned}
& g^{\prime}(z)=\left(z+\frac{1}{2}\right)\left(-1+\left(z^{2}+z\right)^{1 / 2} \log \frac{z+1}{z}\right), \\
& h^{\prime}(z)=\left(z+\frac{1}{2}\right)\left[-1+\left(z+\frac{1}{2}\right) \log \frac{z+1}{z}\right] .
\end{aligned}
$$

We must next find the values (42) which these functions take along the negative real axis. Of course the imaginary components $v_{o}^{\prime}$ and $v_{h}^{\prime}$ are given by (45). The real components are readily found to be

$$
\left\{\begin{array}{l}
u_{o}^{\prime}=\left(\frac{1}{2}-x\right)\left[\left(-1+\pi\left(x-x^{2}\right)^{1 / 2}\right]\right. \\
u_{h}^{\prime}=\left(\frac{1}{2}-x\right)\left[-1+\left(\frac{1}{2}-x\right) \log \frac{1-x}{x}\right] .
\end{array}\right.
$$

Substituting (45) and (46) in (43), and remembering that $r=1$, we obtain

$$
\begin{aligned}
& \pi \frac{d \Phi_{0}(x)}{d x}=\pi\left(\frac{1}{2}-x\right)(1-x) \\
& \quad+\left[M-\left(\frac{1}{2}-x\right)+x\left(\frac{1}{2}-x\right) \log \frac{1-x}{x}\right]\left(\frac{1-x}{x}\right)^{1 / 2} \\
& \frac{d \Phi_{h}(x)}{d x}=\pi\left(\frac{1}{2}-x\right)^{2} \\
& \quad+\left[M-\left(\frac{1}{2}-x\right)+\left(\frac{1}{2}-x\right)^{2} \log \frac{1-x}{x}\right]\left(\frac{1-x}{x}\right)^{1 / 2}
\end{aligned}
$$


Our problem now is to choose $M$ so that these $\Phi$ 's will be monotonic non-decreasing in the interval $0 \leqq x \leqq 1$. Inspection shows that this can be done by setting $M=1 / 2$.

Using this value of $M$ we readily find from (5) that the corresponding functions, which we will call $g(z) / q_{2}$ and $h(z) / q_{1}$, are

$$
\begin{aligned}
\frac{g(z)}{q_{2}}= & \left(z+\frac{1}{2}\right)\left(z+\frac{1}{2}-\left(z^{2}+z\right)^{1 / 2}\right) \log \frac{z+1}{z} \\
& +z-\left(z^{2}+z\right)^{1 / 2}, \\
\frac{h(z)}{q_{1}} & =\left(z+\frac{1}{2}\right)^{2}\left[1+\left(\frac{z+1}{z}\right)^{1 / 2}\right] \log \frac{z+1}{z} \\
& -z-1-\left(z^{2}+z\right)^{1 / 2} .
\end{aligned}
$$

Both of these functions, then, have Stieltjes expansions.

But by (44) and (35),

$$
z=\frac{p^{2}}{2 p+1}, \quad q_{1}=\frac{p}{2 p+1}, \quad q_{2}=p .
$$

Hence

$$
G(p) \equiv g(z)=\frac{2 p^{2}+2 p}{(2 p+1)^{2}}\left(p^{2}+p+\frac{1}{2}\right) \log \frac{p+1}{p}-\frac{p^{2}}{2 p+1}
$$

and

$$
\begin{gathered}
H(p) \equiv h(z)=\frac{2 p^{2}+2 p+1}{(2 p+1)^{2}}\left(p^{2}+p+\frac{1}{2}\right) \log \frac{p+1}{p} \\
-\frac{p^{2}+p}{2 p+1}
\end{gathered}
$$

must have expansions in the form (39).

We see at once that

$$
F^{*}(p)=G(p)+H(p)+p,
$$


the extra term $p$ being an entire function which generates a zero $\Phi(x)$. It occurs because $F^{*}(p)$ has a pole at $\infty$.

This result is an entirely satisfactory one from the standpoint of design, for not only $G(p)$ and $H(p)$, but $\Delta(p) \equiv p$ as well, are constructible, the resultant structure consisting of an inductance and two ladder networks in series.

Bell Telephone Laboratories, New York City

\section{A CORRECTION}

By E. T. BELL

Dr. M. A. Basoco has kindly pointed out to me that the isomorphism established in my paper in the last issue of this Bulletin, ${ }^{*}$ is not, as there stated, partial, but complete. In the second sentence of $\$ 3$, page 323 , the word not should be deleted. Obviously the relation stated is identical with (B). On page 324 (8), the words but not should be replaced by the word and. The error arose from replacing a certain intermediary function of my first draft by $\lambda(z)$; the discarded function did not satisfy (B), but it failed to give a satisfactory analogy in some other respects.

As the matter now stands, it is clear that more is proved than was stated in my paper: my revised Lucas functions are identical with the stated Weierstrassian normal forms having the given invariants. Complete isomorphism is possible, provided we attend in Halphen's theorem to the degenerate case of one period infinite.

* A partial isomorphism between the functions of Lucas and Weierstrass, this Bulletin, vol. 35 (1929), pp. 321-325. 